\title{
Influence of hypertension, left ventricular hypertrophy, and left ventricular systolic dysfunction on plasma $\mathrm{N}$ terminal proBNP
}

\author{
S Talwar, A Siebenhofer, B Williams, L Ng
}

\begin{abstract}
Objectives-To examine the relation between plasma concentration of the $\mathrm{N}$ terminal of the precursor of brain natriuretic peptide (NT proBNP), left ventricular hypertrophy (LVH), and left ventricular systolic dysfunction (LVSD) in patients with a history of hypertension.

Design-Prospective study.

Setting-Teaching hospital based study.

Patients-NT proBNP concentrations were determined in five groups of individuals. Group 1: 15 echocardiographic normal controls; group 2: 22 patients with hypertension, normal left ventricular systolic function, and no LVH; group 3: 24 patients with hypertension, normal left ventricular systolic function, and LVH; group 4: 13 patients with history of hypertension, no history of ischaemic heart disease, and left ventricular wall motion index (WMI) between 1.9-1.3; and group 5:17 patients with a history of hypertension, no history of ischaemic heart disease, and WMI $<1.2$.

Results-Median (range) NT proBNP concentrations (in fmol/ml) for groups 1-5, respectively, were: 129.4 (53.6-159.7), 147.4 (54.3-730.5), 137.1 (35.8-403.9), 356.7 (124.4-934.4), and 493.5 (248.9-909). Mean log NT proBNP differed among all five groups $(\mathrm{p}<0.0001)$, and between groups 4 and 5 versus groups $1-3(p<0.0001)$, and group 4 versus group $5(p=0.02)$ only.

Conclusions-The results suggest that the presence of hypertension with or without LVH (and normal left ventricular systolic function) does not affect NT proBNP concentrations. Moreover, there is a significant rise in NT proBNP only when LVSD develops in hypertension. Thus, NT proBNP remains a useful diagnostic aid for LVSD, even in hypertensive patients.

(Heart 2000;83:278-282)
\end{abstract}

Keywords: hypertension; brain natriuretic peptide; left ventricular hypertrophy; left ventricular systolic dysfunction; chemiluminescence

\section{Department of Medicine and Therapeutics, Robert Kilpatrick Clinical Sciences Building, Leicester Royal Infirmary, Leicester LE2 7LX, UK \\ $S$ Talwar \\ L L Ng \\ Cardiovascular Research Institute, University of Leicester, \\ Leicester, UK \\ A Siebenhofer \\ B Williams \\ Correspondence to: Dr Ng email: LLN1@1e.ac.uk}

Accepted 25 October 1999
Brain natriuretic peptide (BNP) is a 32 amino acid peptide that is synthesised predominantly in the left ventricle as the 108 amino acid prohormone prepro-BNP $(\gamma \mathrm{BNP}) .^{1{ }^{2}}$ The hormone is a potent vasodilator and natriuretic factor that regulates salt and water homeostasis. It has been shown that plasma BNP concentrations are increased in heart failure and following a myocardial infarction, and that in these conditions the concentrations are good indices of prognostic outcome. ${ }^{34}$ In addition, several reports have shown that plasma BNP concentrations are also higher in patients with essential hypertension compared with normotensive subjects, despite significant overlap between the two groups. ${ }^{5-8}$ Furthermore it has been shown that plasma BNP is increased to a greater extent in hypertensive patients with left ventricular hypertrophy (LVH) as determined by echocardiography, and in those hypertensives who have evidence of diastolic dysfunction. ${ }^{9} 10$

Recent work has reported the existence of $\mathrm{NT}$ proBNP (an $8.6 \mathrm{kD}$ peptide, the $\mathrm{N}$ terminal 76 amino acids of preproBNP). In a small study of patients with cardiac failure, NT proBNP has been shown to circulate in plasma in higher concentrations than BNP-32. ${ }^{11}$ There was a small but non-significant increase in plasma NT proBNP concentrations in 20 untreated hypertensives when compared to normal subjects. ${ }^{12}$ A recent study comparing NT proBNP with BNP-32 suggested that the measurement of NT proBNP is an equally sensitive indicator of left ventricular dysfunction after acute myocardial infarction when compared to BNP-32. ${ }^{13}$

There has been no study in hypertensive patients looking at the effect of LVH and left ventricular systolic dysfunction (LVSD) on plasma concentrations of NT proBNP. Measurement of NT proBNP in hypertensive patients may give information on the presence of LVH or LVSD just as a glycosylated haemoglobin measurement in diabetic subjects gives information regarding poor glycaemic control. The potential clinical use of this peptide as a marker of LVH and LVSD in hypertensive subjects who may be on treatment is not known.

\section{Method}

SUBJECTS

Forty six consecutive new referrals of hypertensive patients to the Leicester hypertension clinic, in whom measurements of left ventricular mass were obtainable ( 23 men, median age 44 years, range $22-74$ years), were studied after informed written consent for participation in the study was obtained. Hypertension in the 
untreated group was defined as a mean day systolic blood pressure $>140 \mathrm{~mm} \mathrm{Hg}$, mean diastolic blood pressure $\geqslant 90 \mathrm{~mm} \mathrm{Hg}$, or both, on 24 hour ambulatory blood pressure monitoring (ABPM). Eleven patients in the study were not receiving any antihypertensive treatment. The remaining patients were continued on antihypertensive medication during the study. Exclusion criteria were: a past history of myocardial infarction or heart failure; acute myocardial infarction; active ischaemia; left ventricular dysfunction as defined by fractional shortening $<25 \%$ or the presence of one or more hypokinetic/akinetic segments on echocardiography (wall motion index (WMI) < 2); or renal failure (plasma creatinine concentrations $>170 \mu \mathrm{mol} / \mathrm{l})$.

A further 30 patients $(20$ men, median age 72.5 years, range $43-91$ years) with a history of hypertension (but no history of ischaemic heart disease) and LVSD (median WMI 1.1, range 0.2-1.9) were recruited.

In addition 15 echocardiographically normal controls (nine men, median age 40 years, range 25-76 years, normotensive, no drug treatment, no systolic dysfunction on echocardiography, and no significant valvar abnormality on echocardiography) were studied. This group was used to obtain the normal range in the study.

\section{ECHOCARDIOGRAPHY}

M mode echocardiography was obtained on the 46 consecutive hypertensive patients by two dimensional monitoring using a Hewlett Packard Sonos 1500 imaging system and recorded on super VHS tapes. The patients were examined in the partial left lateral position. Left ventricular chamber recordings were obtained at the tip of the mitral valve. Interventricular septal thickness (IVST) and posterior wall thickness (PWT) were measured at end diastole. Left ventricular internal dimensions (LVID) were obtained at end diastole and at end systole. The measurements were obtained in accordance with the recommendations of the American Society of Echocardiography. ${ }^{14}$

Left ventricular mass was calculated using the equation described by Devereux and Reichek. ${ }^{15}$ Left ventricular mass index (LVMI) was calculated as the ratio of left ventricular mass to body surface area. Upper limits for LVMI at our institution are $125 \mathrm{~g} / \mathrm{m}^{2}$ and $110 \mathrm{~g} / \mathrm{m}^{2}$ for men and women, respectively. Fractional shortening was calculated as the percentage of change in the internal left ventricular dimension between systole and diastole.

Left ventricular WMI, a regional measurement of LVSD which has been shown to be closely correlated to left ventricular ejection fraction (LVEF) by radionuclide cardiography and invasive ventriculography, was calculated using a nine segment model originally described by Heger and colleagues for all the patients. ${ }^{16-18}$ The scale used for WMI has been validated and a linear correlation with $\mathrm{LVEF}$ demonstrated. ${ }^{19}{ }^{20} \mathrm{WMI}$ multiplied by 0.3 gives an estimate of LVEF. ${ }^{16}$ 24 HOUR AMBULATORY BLOOD PRESSURE

The ABPM recordings were obtained using Spacelabs 90207 ABP monitors (Berkshire, UK). Systolic and diastolic blood pressures were recorded at 15 minute intervals during the day (07:00 to $22: 00)$ and at 30 minute intervals during the night (22:00 to 07:00).

BLOOD SAMPLES

A $20 \mathrm{ml}$ sample of venous blood was taken at the time of the echocardiography in all patients. The blood was transferred into prechilled EDTA $(1.5 \mathrm{mg} / \mathrm{ml}$ blood $)$ tubes containing $500 \mathrm{IU} / \mathrm{ml}$ of aprotinin. Samples were immediately centrifuged and plasma separated and then stored at $-70^{\circ} \mathrm{C}$ until assayed.

IMMUNOLUMINOMETRIC ASSAY FOR NT PROBNP Our methodology for the assay of NT proBNP has been described previously. ${ }^{21}$ Briefly, we used a sensitive and specific, non-radioactive immunoluminometric (ILMA) assay based on competitive ligand binding. Stored plasma samples were acidified with $1 \%$ trifluoroacetic acid (TFA) and loaded onto $\mathrm{C}_{18}$ cartridges (Peninsular Laboratories, Merseyside, UK) and eluted with $1 \%$ TFA containing $60 \%$ acetonitrile. The samples were then lyophilised in a centrifugal evaporator, and redissolved in assay buffer consisting of $0.1 \mathrm{~mol} / 1$ sodium phosphate $(\mathrm{pH} 7.4), 0.1 \%$ Triton $\mathrm{X}-100$ for measurement of NT proBNP. The chemiluminescent label 4-(2-succinimidyloxycarbonyl ethyl) phenyl-10-methylacridinium 9-carboxylate fluorosulfonate (Molecular Light Technology, Cardiff, UK) was used to label the peptide representing a domain in the $\mathrm{C}$ terminal section of NT proBNP (amino acids 65-76). NT proBNP concentrations were determined blind to patient details. The normal range for the assay is $<200 \mathrm{fmol} / \mathrm{ml}$.

STATISTICAL ANALYSIS

Concentrations of NT proBNP, clinic blood pressure recordings, ABPM recordings, creatinine, left ventricular mass, IVST, and PWT were not normally distributed and were log transformed before analysis. All results are expressed as median (range) and comparisons were by the Mann Whitney test or Student's $t$ test for unpaired data. Pearson's correlation coefficients were also computed. All statistical analyses were carried out using the software package Minitab (Minitab, Pennsylvania, USA). Comparisons with $\mathrm{p}<0.05$ were considered significant.

\section{Results}

Table 1 shows the clinical characteristics, clinic blood pressure readings, ABPM analysis, echocardiographic data, and serum creatinine measurements of the hypertensive patients with and without LVH. Table 2 shows the antihypertensive drug treatment received by the hypertensive group with and without LVH.

Among the hypertensives with no LVH only six patients were not receiving antihypertensive drug treatment. Eleven patients were taking 
Table 1 Clinical characteristics of the hypertensive patients with and without LVH

\begin{tabular}{|c|c|c|c|c|c|}
\hline & \multicolumn{2}{|c|}{$\begin{array}{l}\text { Hypertension without } L V H \\
(n=22)\end{array}$} & \multicolumn{2}{|c|}{$\begin{array}{l}\text { Hypertension with LVH } \\
(n=22)\end{array}$} & \multirow[b]{2}{*}{$p$ Value } \\
\hline & Median & Range & Median & Range & \\
\hline Age (years) & 40 & $22-65$ & 50 & $28-74$ & $\mathrm{p}=0.02$ \\
\hline Clinic systolic blood pressure ( $\mathrm{mm} \mathrm{Hg}$ ) & 149 & $132-204$ & 165 & $130-226$ & $\mathrm{p}=0.03$ \\
\hline Clinic diastolic blood pressure $(\mathrm{mm} \mathrm{Hg})$ & 98 & $80-112$ & 97 & $76-118$ & $\mathrm{p}=0.78$ \\
\hline Mean day systolic ABPM readings ( $\mathrm{mm} \mathrm{Hg}$ ) & 140 & $122-179$ & 152 & $125-176$ & $\mathrm{p}=0.02$ \\
\hline Mean day diastolic ABPM readings $(\mathrm{mm} \mathrm{Hg}$ ) & 90.5 & $74-104$ & 92 & $71-112$ & $\mathrm{p}=0.32$ \\
\hline Mean night systolic ABPM readings ( $\mathrm{mm} \mathrm{Hg}$ ) & 127.5 & $106-146$ & 133 & $100-218$ & $\mathrm{p}=0.26$ \\
\hline Mean night diastolic $\mathrm{ABPM}$ readings $(\mathrm{mm} \mathrm{Hg})$ & 77 & $61-96$ & 76.5 & $57-134$ & $\mathrm{p}=0.87$ \\
\hline Creatinine $(\mathrm{mmol} / \mathrm{l})$ & 81 & $47-165$ & 74 & $55-142$ & $\mathrm{p}=0.47$ \\
\hline $\operatorname{LVMI}\left(\mathrm{gm} / \mathrm{m}^{2}\right)$ & 98 & $68-121$ & 134 & $115-222$ & $\mathrm{p}<0.0001$ \\
\hline $\operatorname{IVST}(\mathrm{cm})$ & 0.97 & $0.81-1.14$ & 1.17 & $0.87-1.62$ & $\mathrm{p}=0.0001$ \\
\hline PWT (cm) & 0.96 & $0.75-1.15$ & 1.15 & $0.84-1.64$ & $\mathrm{p}=0.0002$ \\
\hline Fractional shortening (\%) & 46 & $28.7-55.8$ & 39.4 & $25.3-65.6$ & $\mathrm{p}=0.27$ \\
\hline
\end{tabular}

Table 2 Antihypertensive drug treatment of hypertensive patients with and without LVH

\begin{tabular}{lll}
\hline & $\begin{array}{l}\text { Hypertension } \\
\text { with no LVH }\end{array}$ & $\begin{array}{l}\text { Hypertension } \\
\text { with LVH }\end{array}$ \\
\hline Thiazide & 6 & 9 \\
$\beta$ Blocker & 2 & 7 \\
Calcium channel blocker & 4 & 9 \\
ACE inhibitor & 6 & 8 \\
a Blocker & 4 & 1 \\
Angiotensin II blocker & 1 & 4 \\
Other & 1 & 2 \\
\hline
\end{tabular}

one drug, four patients were on two drugs, and one patient was on four different antihypertensive agents. In the group with LVH five patients were on no antihypertensive drug treatment, five patients were taking one drug, nine patients were on two drugs, three patients were on three drugs, and two patients were on four different antihypertensive agents. The patients with $\mathrm{LVH}$ were on a significantly larger number of antihypertensive drugs $(\mathrm{p}<0.01)$.

The clinic systolic blood pressure was correlated to the mean day and night time systolic readings on the ABPM $(r=0.81$ and $r=0.70$, respectively, both $\mathrm{p}<0.001)$. Also the clinic diastolic blood pressure was correlated to the mean day and night time diastolic readings on the ABPM $(r=0.76$ and $r=0.70$, respectively, both $\mathrm{p}<0.001)$.

The LVMI was correlated to the age of the patient $(r=0.37, \quad \mathrm{p}=0.01), \quad \log$ IVST $(r=0.77, \mathrm{p}<0.001), \log$ PWT $(r=0.74$, $\mathrm{p}<0.001)$, and to $\log$ transformed clinic systolic blood pressure $(r=0.30, \mathrm{p}=0.04)$.

Table 3 shows the clinical characteristics, echocardiographic data, and serum creatinine measurements of the hypertensive patients with LVSD.

In those patients with a WMI $>1.3$ only four gave a history of shortness of breath and three had clinical evidence of left ventricular failure. Six of these patients were on a diuretic and three were on an angiotensin converting enzyme (ACE) inhibitor. In the group with a WMI < 1.2, 14 patients gave a history of shortness of breath and 13 had clinical evidence of left ventricular failure. In this group 11 were on a diuretic and 10 were on an ACE inhibitor.

Table 4 shows the NT proBNP concentrations for the study population. Mean log NT proBNP differed among all five groups ( $p<0.0001$, analysis of variance) and between groups 4 and 5 versus groups 1-3 $(\mathrm{p}<0.0001)$, and group 4 versus group 5 $(\mathrm{p}=0.02)$ only (fig 1$)$. The patients in groups 4 and 5 were older than those in groups 1-3 $(\mathrm{p}<0.001)$. There was no significant difference in ages between group 1 versus group 2, and group 1 versus group 3 . The subjects in group 3 were older than those in group 2, however.

There was no significant correlation between $\log$ NT proBNP in the hypertensive patients (with normal systolic function) and the clinic blood pressure measurements or the ABPM recordings. There was also no significant correlation in this group between log NT proBNP and LVMI, log IVST, log PWT, age, or $\log$ creatinine.

On multiple regression analysis for the hypertensives without LVSD (groups 2 and 3), the age of the patient was independently associated with LVMI $\left(\mathrm{R}^{2}=8 \%, \mathrm{p}<0.05\right)$. Log NT proBNP was not a significant predictor of LVMI in the model.

In the group with LVSD there was a significant correlation between log NT proBNP and left ventricular systolic function as assessed by a WMI score $(r=-0.36, \mathrm{p}<0.05)$. The sensitivity, specificity, positive predictive values, and negative predictive values for NT proBNP concentration $>245 \mathrm{fmol} / \mathrm{ml}$ in picking up a WMI score of $<1.2$ in this study population is $100 \%, 74.3 \%, 47.2 \%$, and $100 \%$, respectively.

\section{Discussion}

LVH is known to be an independent risk factor for all the cardiovascular complications of hypertension..$^{22}$ Hence, its early detection is very important in the management of the hypertensive patient. Electrocardiography is recommended for the assessment of every hypertensive patient. Its sensitivity and specificity for detecting LVH is poor, however. ${ }^{23}$ Echocardiography is more sensitive and specific than ECG for the diagnosis of LVH, but it is not practical to perform echocardiography on all patients with hypertension. ${ }^{23}$ As LVH cannot be reliably predicted from blood pressure levels, a non-invasive and inexpensive method for the detection of LVH and poor blood pressure control in hypertensive patients would be clinically useful. ${ }^{24}$

The present study examined the ability of a competitive binding immunoluminometric assay for NT proBNP to detect LVH or LVSD in hypertensive patients. Antihypertensive or 


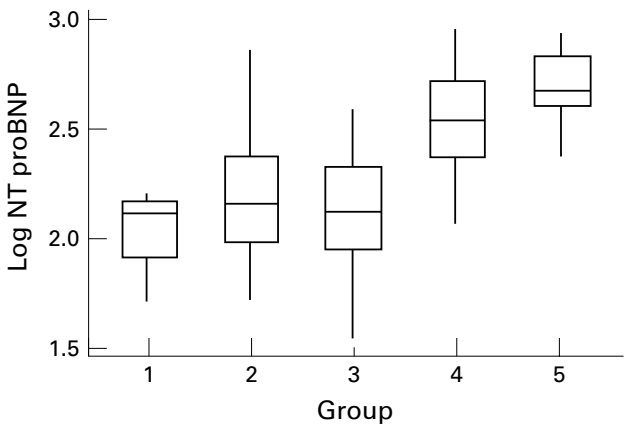

Figure 1 Box plot showing the relation between $\log$ NT proBNP concentrations and echocardiographic normal controls (group 1), patients with hypertension, normal left ventricular systolic function, and no LVH (group 2), patients with hypertension, normal left ventricular systolic function, and LVH (group 3), patients with a history of hypertension and left WMI between 1.9-1.3 (group 4), and patients with a history of hypertension and WMI of $<1.2$ (group 5)

anti-heart failure treatment was not withheld in these patients because it was felt that if NT proBNP is to be used as a clinical tool in detecting high risk hypertensive patients it is best tested in patients who are receiving treatment. Moreover, it was considered unethical to withdraw treatment in poorly controlled hypertensive subjects.

In the present study we have shown that plasma NT proBNP concentrations are raised in hypertensive subjects with established LVSD when compared to normotensive normal controls. There was no significant difference between the NT proBNP concentrations in the hypertensives with or without $\mathrm{LVH}$ and the controls. This is in broad agreement with the published data on NT proBNP ${ }^{12}$ where a competitive radioimmunoassay for the $\mathrm{N}$ terminal of NT proBNP was used (in contrast to the assay for the $\mathrm{C}$ terminal of NT proBNP used in the current study), and for BNP as measured by radioimmunoassay. ${ }^{5-8}$ Although there was a trend towards possibly higher NT proBNP concentrations in the hypertensive group without LVSD, the study was probably inadequately powered to reveal a significant difference.

We have shown that NT proBNP concentrations can be used to detect LVSD in hypertensive subjects, even in the absence of clinical symptoms and signs of heart failure. Only 18 of the 30 subjects with LVSD gave a history of

Table 3 Clinical characteristics of the hypertensive patients with LVSD

\begin{tabular}{lcccccc}
\hline & \multicolumn{2}{l}{$W M I \geqslant 1.3(n=13)$} & & \multicolumn{2}{l}{$W M I \leqslant 1.2(n=17)$} & \\
\cline { 2 - 3 } & Median & Range & & Median & Range & p Value \\
\hline Age (years) & 75 & $56-91$ & & 66 & $43-83$ & $\mathrm{p}=0.1$ \\
Creatinine (mmol/1) & 102 & $74-144$ & & 107.5 & $68-285$ & $\mathrm{p}=0.7$ \\
WMI & 1.6 & $1.3-1.9$ & & 0.6 & $0.2-1.2$ & $\mathrm{p}<0.0001$ \\
\hline
\end{tabular}

Table 4 NT proBNP levels for the study population

\begin{tabular}{ll}
\hline Study groups & $\begin{array}{l}\text { Median (range) concentration NT } \\
\text { proBNP (fmol/ml) }\end{array}$ \\
\hline Group 1: normal controls & $129.4(53.6-159.7)$ \\
Group 2: hypertensives with no LVH & $147.4(54.3-730.5)$ \\
Group 4: hypertensives with LVH & $137.1(35.8-403.9)$ \\
Group 4: hypertensives with WMI $\geqslant 1.3$ & $356.7(124.4-934.4)$ \\
Group 5: hypertensives with WMI $\leqslant 1.2$ & $493.5(248.9-909.0)$ \\
\hline
\end{tabular}

shortness of breath and only 16 of the 30 subjects had evidence of clinical left ventricular failure. This may be of importance clinically as it may determine the type of antihypertensive treatment that may be used. This may lead to increased use of ACE inhibition in patients with subclinical LVSD which may ultimately improve their prognosis, although this would obviously have to be tested by a prospective study.

In our study plasma NT proBNP concentrations were not significantly correlated to left ventricular mass, LVMI, IVST, PWT, and mean arterial pressures. Plasma BNP concentrations in a previous study had correlated to IVST, PWT, and LVMI ${ }^{9}$; however, antihypertensive treatment was discontinued at least a week before the commencement of that study. It is well recognised that antihypertensive drug treatment can regress $\mathrm{LVH}$ and also decrease the concentrations of the natriuretic peptides in the plasma. ${ }^{25}$ Thus, it is possible that antihypertensive drug treatment may have influenced the results in our study and accounted for the lack of correlation between NT proBNP concentrations and the LVMI or the degree of hypertrophied wall thickness. In our study the hypertensive patients with $\mathrm{LVH}$ were receiving a significantly larger number of antihypertensive drugs. Other explanations for the lack of correlation between NT proBNP and echocardiographic parameters are unlikely. The peptide assay is both reliable and accurately reproducible in our hands. ${ }^{21}$

We did not attempt to assess diastolic dysfunction by echocardiography because a phenomenon as complex as diastolic dysfunction cannot be accurately assessed by simple Doppler indices. ${ }^{26}$ Some patients in group 2 and 3 had notably increased concentrations of NT proBNP. It is certainly possible that diastolic dysfunction in these patients may have contributed to high peptide concentrations. ${ }^{9} 10$

We have included patients with LVSD, a history of hypertension, and no history of ischaemic heart disease, thus implying that the LVSD observed in the study was caused by hypertension and probably not by ischaemic heart disease. It must, however, be emphasised that silent ischaemia is not uncommon and ischaemic heart disease may therefore be the primary cause of LVSD in these patients. Active exclusion of patients by means of a coronary angiogram was felt unnecessary and would have proved difficult to do practically.

In conclusion, this cross sectional study has shown that plasma NT proBNP concentrations are increased in hypertensive patients with established LVSD and not when LVH is present along with normal systolic function. We have shown for the first time that raised NT proBNP concentrations in a hypertensive population reflects LVSD but does not add any further clinical information about LVH. The test thus remains a useful aid for the detection of LVSD even in hypertensive patients. We thank the Leicester Royal Infirmary for ST's support, and
the staff in the cardiology services department and the Leicester the staff in the cardiology services department and the Leicester
Hypertension Clinic for their help and support. We also thank Hypertension Clinic for their help and support. We also thank
Ms Paulene Quinn and Sonja Jennings for excellent technical 
assistance, and Stuart Woodhead and Ian Weeks of Molecular Light Technology for the gift of the methyl acridinium ester.

1 Tateyama H, Hino J, Minamino N, et al. Characterization of immunoreactive brain natriuretic peptide in human cardiac immunoreactive brain natriuretic peptide in human cardiac

2 Yandle TG, Richards AM, Gilbert A, et al. Assay of brain Yandle TG, Richards AM, Gilbert A, et al. Assay of brain
natriuretic peptide (BNP) in human plasma: evidence for natriuretic peptide (BNP) in human plasma: evidence for high molecular weight BNP as a major plasma compone

3 Yoshimura M, Yasue H, Okumura K, et al. Different secretion patterns of atrial natriuretic peptide and brain natriuretic peptide in patients with congestive heart failure. Circulation 1993; 87:464-9.

4 Omland T, Aakvaag A, Bonarjee VVS, et al. Plasma brain natriuretic peptide as an indicator of left ventricular systolic function and long-term survival after acute myocardia infarction. Circulation 1996;93:1963-9.

5 Buckley MG, Markandu ND, Miller MA, et al. Plasma concentrations and comparisons of brain and atrial natriuretic peptide in normal subjects and in patients with essential hypertension. F Hum Hypertens 1993;7:245-50.

6 Kohno M, Horio T, Yokokawa K, et al. Brain natriuretic peptide as a cardiac hormone in essential hypertension. Am peptide as a cardiac hor
$\mathcal{F}$ Med $1992 ; \mathbf{9 2}: 29-34$.

7 Naruse M, Takeyama Y, Taanabe A, et al. Atrial and brain natriuretic peptides in cardiovascular disease. Hypertension 1994:23 (suppl I):231-I-4-I.

8 Mukoyama M, Nakao K, Saito Y, et al. Human brain natriuretic peptide, a novel cardiac hormone. Lancet 1990;335 $801-2$.

9 Nishikimi T, Yoshihara F, Morimoto A, et al. Relationship between left ventricular geometry and natriuretic peptide levels in essential hypertension. Hypertension 1996;28:2230.

10 Cheung BM. Plasma concentrations of brain natriuretic peptide is related to diastolic dysfunction in hypertension. Clin Exp Pharmacol Physiol 1997;24:966-8.

11 Hunt PJ, Yandle TG, Nicholls MG, et al. The aminoterminal portion of pro-brain natriuretic peptide (proBNP) circulates in human plasma. Biochem Biophys Res Commun 1995;214:1175-83.

12 Hunt PJ, Richards AM, Nicholls MG, et al. Immunoreactive amino-terminal pro-brain natriuretic peptide (NTproBNP): a new marker of cardiac impairment. Clin EndoproBNP): a new marker
crinol 1997;47:287-96.

13 Richards AM, Nicholls MG, Yandle TG, et al. Plasma N-terminal pro-brain natriuretic peptide and ventricular function and prognosis after myocardial infarction. Circulation 1998;97:1921-9.

14 Sahn DJ, DeMaria A, Kisslo J, et al. Recommendations regarding quantitation im $\mathrm{M}$-mode echocardiography: results of a survey of echocardiographic measurements. Circulation 1978;58:1072-83.

15 Devereux RB, Reichek N. Echocardiographic determination of left ventricular mass in man. Circulation 1977;55:61318

16 Berning J, Rokkedal Nielsen J, Launbjerg J, et al. Rapid estimation of left ventricular ejection fraction in acute myocardial infarction by echocardiographic wall motion analysis. Cardiology 1992;80:257-66.

17 Rifkin RD, Koito H. Comparison with radionuclide angiography of two new geometric and four non-geometric models for echocardiographic estimation of left ventricular ejection fraction using segmental wall motion scoring. Am f Cardiol 1990;65:1485-90.

18 Heger JJ, Weyman AE, Wann LS, et al. Cross-sectional echocardiographic analysis of the extent of left ventricular asynergy in acute myocardial infarction. Circulation 1980; 61:113-18.

19 Berning J, Steensgaard-Hansen F. Early estimation of risk by echocardiographic determination of wall motion index in an unselected population with acute myocardial infarction. Am f Cardiol 1990;65:567-76.

20 Kober L, Torp-Pederson C, Carlsen J, et al. An echocardiographic method for selecting high risk patients shortly after acute myocardial infarction, for inclusion in multicentre studies. Eur Heart f 1994;15:1616-20.

21 Hughes D, Talwar S, Squire IB, et al. An immunoluminometric assay (ILMA) for N-terminal proBNP: development of a test for left ventricular dysfunction. Clin Sci 1999;96:373-80.

22 Levy D, Garrison RJ, Savage DD, et al. Prognostic implications of echocardiographically determined left ventricular mass in the Framingham heart study. N Engl f Med 1990; 322:1561-6.

23 Devereux RB, Casale PN, Eisenberg RR, et al. Electrocardiographic detection of left ventricular hypertrophy using echocardiographic determination of left ventricular mass as the reference standard: comparison of standard criteria, computer diagnosis and physician interpretation. $7 \mathrm{Am}$ Coll Cardiol 1984;3:82-7.

24 Frohlich ED, Apstein C, Chobanian AV, et al. The heart in hypertension. N Engl f Med 1992;327:998-1008.

25 Kohno M, Yokokawa K, Yasunari K, et al. Changes in plasma cardiac natriuretic peptide concentrations during 1 year treatment with angiotensin-converting enzyme inhibitor in elderly hypertensive patients with left ventricular hypertrophy. Int f Clin Pharmacol Ther 1997;35:38-42.

26 Anon. ACC/AHA guidelines for the clinical application of echocardiography: a report of the American College of Cardiology/American Heart Association task force on practical guidelines. Circulation 1997;95:1686-744. 